\title{
A Novel Approach for Placement of Phasor MEASUREMENT UNIT \& COUNTING THEIR OPTIMAL NUMBER
}

\author{
Ajit Kumar and S Bhongade
}

Department of Electrical Engineering, S G S Institute of Technology \& Science, Indore452003, M.P-INDIA

\begin{abstract}
In India Power system monitoring is based mainly on measurements provided by Remote Terminal Unit (RTU). For power system measurement synchronized Phasor or Phasor Measurement Unit (PMU) is popular choice in abroad and its popularity is increasing day by day because of its several features. Direct measurement of voltage or current Phasor is possible because of PMU which was not possible earlier. Global positioning system (GPS) provides time synchronized data. In this paper a new approach is used for optimal placement of PMU, counting their number \& time elapsed. This approach includes zero injection method along with single hop connectivity. The approach is successfully applied on IEEE-14 bus system. Here all the bus which are taken into consideration is either completely observable or at some depth of UnObservability is also taken into account.
\end{abstract}

\section{KEYWORDS}

PMU, Observability, Phasor, Zero injection.

\section{INTRODUCTION}

The load demand in power system is always varying during whole day. The minimum capacity of generator should be such that it should meet the maximum demand. Stable operation of power system requires accurate and online monitoring of various operating conditions. A conventional method of power system monitoring is accomplished by SCADA (Supervisory Control and Data Acquisition system) system .SCADA system usually consists of Remote Terminal Units (RTU). RTUs are the microprocessor controlled electronic device which can measure real and reactive power flow, magnitude of bus voltage and current. RTUs are placed at various substation and they send the measured values to the state estimator which is placed inside the central control center. From the received measurement values and the knowledge about the network topology, state estimator can estimate various electrical quantities related to the power system stability. These estimated values are used for the online power flow control and management. But one of the drawbacks of the RTU is that it cannot directly measures the phase angle of the voltage and current at any bus. If we can measure Phasor values of bus voltages and currents, better state estimation and thereby better power system control can be achieved. With the aid of Global Positioning System (GPS), a new era of measurement technique was developed in mid-1980s 
called Phasor Measurement Units (PMU). PMU utilizes the synchronization signal from the GPS to provide the Phasor values of voltage and current at the bus or substation where it is connected [1]. These types of Phasor measurements can improve the monitoring of power system. PMUs can measure the phase angle and amplitude of voltage at the installed bus and current through all branches connected to that bus but it is redundant and not economical to place PMUs at all buses for making the power system completely observable. Finding the optimal location for PMU is of great interest so that with minimum number of PMU the power system under consideration will be fully observable [2], [3], [5] \& [6]. The synchrophasor technology was first introduced in India [7],[8] \& [9] in 2010-2011 through a pilot project in the northern region, and subsequently, PMUs have also been placed in some selected stations in southern and western regions of the Indian power grid. Nikolaos $M$ et al [10] presents an optimal placement of PMU based on a quadratic minimization problem with continuous decision variables subject to nonlinear observability constraints. Authors in ref. [11] discussed the smart grid associated technical, environmental and socio-economic effect to society, and the solutions for the challenges of designing and building the future of the power system. Smart Grid (SG) integrated with communication infrastructures and forms an intelligent electricity network working with all connected components to deliver sustainable electricity supplies [12]. Many advanced communication technologies have been identified for SG applications and has been discussed in [12] efficiency of power grids. A non-linear programming based model for optimal placement of PMU has been discussed in [13] to minimize number of PMU units required for full system observability. A Genetic Algorithm based optimized PMU-location-decision algorithm considering WAMS reliability has been proposed in [14]. The proposed algorithm computes the availability of PMU using Markov chains than in order to find the optimal deployment of PMU, the influence of PMU's failure has been analyzed by the indices of WAMS reliability assessment. Authors in [15] present a state estimation method for power systems with incomplete PMU observability. This paper also proposes a model that incorporates time-skew errors in conventional measurements on the performance of hybrid state estimators using both conventional and PMU measurements. This paper proposes the optimal placement for PMUs, counting their number and total time elapsed.

\section{PHASOR MEASUREMENT UNIT}

Fig. 1 shows the functional block diagram of a PMU [1]. PMU measures voltage and phase angle on an electricity grid, using a common time source for synchronization. Analog inputs from potential transformer and current transformer are fed to an anti-aliasing. This will restrict the bandwidth of the signal to approximately satisfy the sampling theorem. The signals from the output of anti aliasing filter are converted to digital using A/D converter. Phase locked loop ensures the synchronization of sampling with reference signal from GPS. 


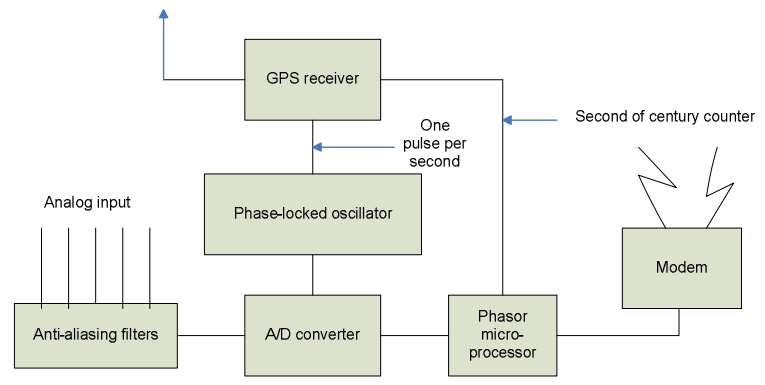

Figure 1: Functional block diagram of PMU.

Sampled signals are then fed to the Phasor microprocessor, where phase voltage and current are computed using recursive Discrete Fourier Transform (DFT) algorithm. The computed of Phasor values are assembled in a message stream and are then sent via the communication network to the wide area monitoring system. Sampled signals are then fed to the Phasor microprocessor, where Phasor of phase voltages and currents are computed using recursive Discrete Fourier Transform (DFT) algorithms. The computed of Phasor value are assembled in a message stream and are then sent via the communication network to the wide area monitoring system.

\section{THE CONCEPT OF OBSERVABILITY}

A bus is said to be observable if a PMU is located on that bus and the voltage and phase angle are measured directly. A bus is said to be indirectly observable, if the voltage Phasor at that bus is estimated using other PMUs. A bus is said to be unobservable, if its voltage Phasor cannot be measured. This condition occurs when there is no PMU at that bus and neighbouring buses. From Fig. 2, at bus 2, $6 \& 9$ a PMU is placed so that voltage magnitude and phase angle at that bus and current through all the branches connected to it can be measured. So the bus 2, $6 \& 9$ are said to be directly observable since PMU is placed on these buses. Voltage magnitude at buses 1, 3, 4, 5, $7,10,11,12,13 \& 14$ can be estimated using ohms law. So these buses are said to be indirectly observable. But the voltage Phasor at bus number 8 cannot be estimated using PMU at any buses, so we can say this bus 8 is unobservable. If all neighbouring buses of an unobservable bus are observable, then it is called depth of one Un-Observability[6] . A power system is said to be completely observable, if all the buses in that system are either directly or indirectly observable. A power system is said to be incompletely observable, if some buses are not observable.

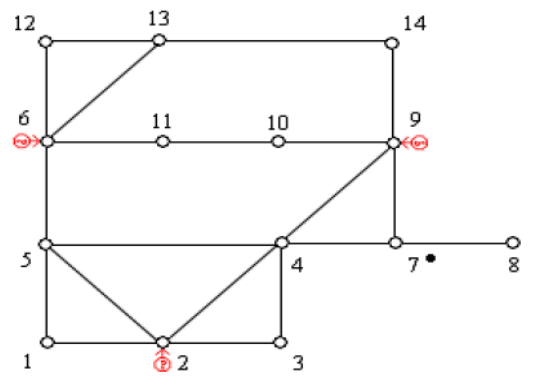

Figure 2: IEEE-14 bus system with P.M.U placement 


\section{BASIC IDEA OF PMU PLACEMENT}

The basic idea of PMU placement is to make entire system observable. For this we have two approach first one is to put PMU at all the substation, which makes entire system cost very high because PMU is a costlier device, the other thing we can do is to optimized the number of PMU. The concept of Observability is defined mathematically as follows:

Let $\mathrm{x}$ be a binary decision vector whose entity is defined as,

$$
x_{i}= \begin{cases}1 & \text { if a } \\ 0 & \text { otherwise }\end{cases}
$$

And

For an n-bus system, the PMU placement can be formulated as;

mentmize $\sum_{i}^{n} w_{i} \cdot x_{i}$

subject to $f(x) \geq 1$

Where $\mathrm{w}$ is the cost of PMU installed at bus i. $\mathrm{f}(\mathrm{x})$ is a vector function, which is non-zero if corresponding bus voltage is solvable or zero otherwise.

\section{Proposed AlgORithM}

As we know that PMU measures bus voltage Phasor and branch current Phasor. Each PMU has certain number of channel to record the value of voltage Phasor and branch current. It is also assumed that each PMU has enough number of channels. We can limit these numbers of channel up to some extent because after reducing their numbers to very small the time elapsed and number of PMU increases above the desirable limit, which is not ideal for our purpose. In this paper a concept of zero injection along with single hop connectivity is used for reducing the number of PMU required for making entire system observable. The figure 2 shows a zero injection at bus 7 . Bus 8 is unobservable because it is not connected with any PMU directly or indirectly so it is not possible to calculate voltage and current here. The concept of zero injection measurement as mentioned in [6] makes the entire system observable and it also reduces the number of PMU by one which was 4 when no zero injection was introduced. The PMU is at bus 2, $6 \& 9$ makes entire system observable only when a zero injection is done at bus 7 . In this paper we have made the entire IEEE-14 bus system observable with the help of $\mathrm{m}$-file coding whose pseudo code is given below. A new concept of zero injection along with single hop connectivity is also defined which is very helpful in achieving the required goal. We have to follow the following six steps written below. The first step of the proposed method completes as we have placed the PMU at those buses which makes entire bus system observable .In second step we have to form a square matrix $\mathrm{A}(14,14)$ from the above placement. 


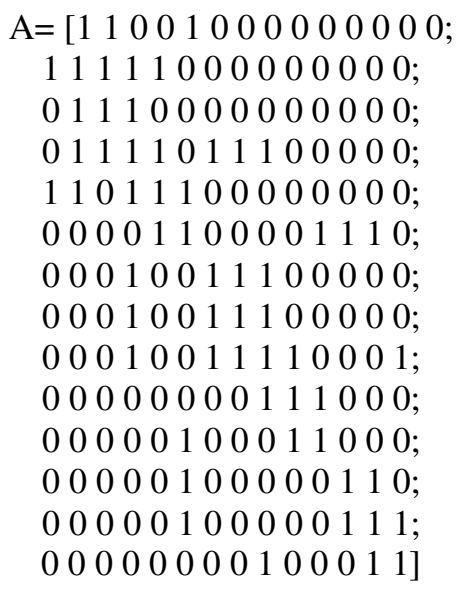

The third step is to make Observability equation from the above matrix which is given below:

$$
f(x)=A . . X=\left\{\begin{array}{l}
f_{1}=x_{1}+x_{2}+x_{5} \geq 1 \\
f_{2}=x_{1}+x_{2}+x_{3}+x_{4}+x_{5} \geq 1 \\
f_{3}=x_{2}+x_{3}+x_{4} \geq 1 \\
f_{4 \_ \text {new }}=x_{2}+x_{3}+x_{4}+x_{5}+x_{7}+x_{8}+x_{9} \geq 1 \\
f_{5}=x_{1}+x_{2}+x_{4}+x_{5}+x_{6} \geq 1 \\
f_{6}=x_{5}+x_{6}+x_{11}+x_{12}+x_{13} \geq 1 \\
f_{7}=x_{4}+x_{7}+x_{8}+x_{9} \geq 1 \\
f_{8 \_ \text {new }}=x_{4}+x_{7}+x_{8}+x_{9} \geq 1 \\
f_{9 \_ \text {new }}=x_{4}+x_{7}+x_{8}+x_{9}+x_{10}+x_{14} \geq 1 \\
f_{10}=x_{9}+x_{10}+x_{11} \geq 1 \\
f_{11}=x_{6}+x_{10}+x_{11} \geq 1 \\
f_{12}=x_{6}+x_{12}+x_{13} \geq 1 \\
f_{13}=x_{6}+x_{12}+x_{13}+x_{14} \geq 1 \\
f_{14}=x_{9}+x_{13}+x_{14} \geq 1
\end{array}\right.
$$

The fourth step is to check whether the whole any redundancy is possible or not. For this check, in fifth step the pseudo code is mentioned. Now check again the Observability of the system, is further any redundancy is possible or not if no stop if yes go again for the fourth step. In this paper for IEEE-14 bus system a zero is injected at bus number 7 because of this all the bus which are adjacent to this bus are modified in the following manner shown above. Here we have consider if a bus is directly connected to zero injection bus it will be modified in the following manner as shown is Observability equation. 


\section{Pseudo CODe}

Input : A an $\mathrm{MxN}$ square matrix ;

Output : disp('The optimum number of PMUs is')

fprintf('\%dln',sum(x))

Initialize $\mathrm{P}=[\mathrm{]}$

for $\mathrm{j}=1: \mathrm{n}$

for $\mathrm{i}=1: \mathrm{j}$

Check condition $A(i, j)=1$;

If $\mathrm{j}=1$

$P(i, j)=1$;

$\mathrm{P}(\mathrm{j}, \mathrm{i})=1$

End first for loop;

End second for loop;

After execution of above code all the diagonal element of $\mathrm{P}$

set to zero.

ChannelLimit = input('Choose a channel limit for the PMUs: ');

Qi = Qout;

Again Initialize $\mathrm{Q}=[]$

for $\mathrm{k}=1: \mathrm{n}$

Check if $\operatorname{sum}(\mathrm{P}(:, \mathrm{k}))<$ Channel Limit

$\mathrm{V}=$ find $(\mathrm{P}(:, \mathrm{k}))$;

$\mathrm{T}=\operatorname{zeros}(1, \mathrm{n})$;

$\mathrm{T}(1, \mathrm{k})=1$;

$\mathrm{T}(1, \mathrm{~V})=1$;

$\mathrm{Q}=[\mathrm{Q} ; \mathrm{T}]$;

If not then execute

$\mathrm{V}=\operatorname{nchoosek}($ find $(\mathrm{P}(:, \mathrm{k}))$, Channel Limit $)$;

$[\mathrm{a}, \mathrm{b}]=\operatorname{size}(\mathrm{V})$;

$\mathrm{T}=\operatorname{zeros}(\mathrm{a}, \mathrm{n})$;

for $\mathrm{i}=1: \mathrm{a}$

for $\mathrm{j}=1: \mathrm{b}$

$\mathrm{T}(:, \mathrm{k})=1$;

$\mathrm{T}(\mathrm{i}, \mathrm{V}(\mathrm{i}, \mathrm{j}))=1$;

end

end

$\mathrm{Q}=[\mathrm{Q} ; \mathrm{T}]$

end

fprintf('\%d $\%$ n',k)

end

Q;

Start Timer

For different bus system like IEEE-14 and IEEE-57

Use injection method as mentioned [3]

Stop timer. 


\section{SIMULATION RESULTS}

A MATLAB program has been developed for the above psedo code and results are given in Table 1. In first column the channel limit required for PMU is shown. Second and third column shows the number of PMU required and time elapsed in second respectively.

Table 1 Channel Limit for PMU.

\begin{tabular}{|c|c|c|}
\hline $\begin{array}{c}\text { Channel limit } \\
\text { for PMU }\end{array}$ & $\begin{array}{c}\text { IEEE-14 Bus } \\
\text { System(number } \\
\text { of PMU) }\end{array}$ & $\begin{array}{c}\text { Time elapsed } \\
\text { in second }\end{array}$ \\
\hline 1 & 7 & 3.286 \\
\hline 2 & 5 & 3.014 \\
\hline 3 & 4 & 0.492 \\
\hline 4 & 3 & 0.059 \\
\hline 5 & 3 & 0.044 \\
\hline
\end{tabular}

\section{CONCLUSIONS}

PMU and their application have brought revolution in wide area monitoring. Optimal placement of PMU is one step towards the concept of smart grid. It can be concluded from the above work that channel limit for PMU is a very important concept because it affects both the total number of PMU used for complete observability as well as time elapsed for its calculation. By optimal placement of PMU we can reduce the overall installation cost as well as make our grid more reliable and smarter. This method can be applicable for higher order bus system like IEEE-30 \& IEEE-57.

\section{REFERENCES}

[1] A. G. Phadke, "Synchronized phasor measurements in power systems", IEEE Computer Applications in Power, Vol. 6, Issue 2, pp. 10-15, April 1993.

[2] A. G. Phadke, J. S. Thorp, and K. J. Karimi, "State Estimation with Phasor Measurements", IEEE Transactions on Power Systems, Vol. 1, No. 1, pp. 233-241,February 1986.

[3] T. L. Baldwin, L. Mili, M. B. Boisen, and R Adapa, "Power System Observability With Minimal Phasor Measurement Placement”, IEEE Transactions on Power Systems, Vol. 8, No. 2, pp. 707- 715, May 1993

[4] R. F. Nuqui and A. G. Phadke, Phasor measurement unit placement based on incomplete observability," in IEEE 2002 Power Engineering Society Summer Meeting, vol. 2, Jul. 25, 2002, pp. 888\{893\}.

[5] Bei Xu, Y. J. Yoon and A. Abur, "Optimal Placement and Utilization of Phasor Measurements for State Estimation”, Power System Computation Conference, August 2005, Liege, Belgium.

[6] Bei Xu and A. Abur, "Observability Analysis and Measurement Placement for Systems with PMUs", Proceedings of the IEEE PES Power Systems Conference and Exposition, October $\quad 2004$, New York, NY.

[7] Hariram,A., "Synchrophasor initiatives in India," Power System Operation Corporation Limited (a wholly owned subsidiary of Power Grid Corporation of India Limited), June 2012, available www.scribd.com/doc/114896908 
Electrical and Electronics Engineering: An International Journal (ELELIJ) Vol 5, No 1, February 2016

[8] Agarwal, V. K., Agrawak, P. K., and Kumar, R., "Experience of commissioning of PMUs pilot project in the northern region of India," 16th National Power Systems Conference "Innovations for Smart Grids, Visakhapatnam, India, 15-17 December 2010.

[9] Ghosh,D.,Ghose, T., and Mohanta,D. K., "Communication feasibility analysis for smart grid with phasor measurement units,” IEEE Trans. Indust. Inform.,Vol. 9,No. 3, pp. 1486-1496, 2013.

[10] Nikolaos M. Manousakis and George N. Korres, "A Weighted Least Squares Algorithm for Optimal PMU Placement”, IEEE Transactions on Power Systems, Vol. 28, No. 3, August 2013.

[11] Mohamed E. El-hawary, "The Smart Grid-State-of-the-art and Future Trends", Electric Power Components and Systems Journal, Vol. No. 42(3-4):pp. 239-250, 2014.

[12] Ma, R., Chen, H.-H., Huang, Y.-R., and Meng, W, "Smart grid communication: Its challenges and opportunities," IEEE Transactions on Smart Grid, Vol. 4, No. 1, pp. 36, 46, March 2013.

[13] Theodorakatos, Nikolaos M. Manousakis and George N. Korres, "Optimal Placement of Phasor Units with Linear and Non-linear Models", Electric Power Components and Systems, Vol. 43, Issue 4, pp. 357-373, 2015.

[14] Xiaowen Huang, Junyong Liu; Yang Liu; Lixiong Xu; Jiashi Yang; Yaqi Ni, “A GA based optimized PMU-location-decision algorithm considering WAMS reliability", in the proceedings of IEEE Conference on Power System Technology (POWERCON) 2014, Pages: 433 - 439, $\quad$ DOI: 10.1109/POWERCON.2014.6993697

[15] Kashyap, N.; Werner, S.; Yih-Fang Huang; Riihonen, T., "Power System State Estimation Under Incomplete PMU Observability-A Reduced-Order Approach", IEEE Transactions on Signal Processing, Vol. 8 Issue No.6, Pages: 1051 - 1062, Dec 2014

\section{AuTHORS}

Ajit Kumar (b’1988) did his master's in Digital Technique and Instrumentation from thedepartment of Electrical Engineering, at S.G.S Institute of Technology \& Science, Indore (M.P)-India. His research interests include Power System and Control, digital system and instrumentation.

Sandeep Bhongade (b'1974) received M.E. degree in Electrical Engineering from V.J.T.I. Mumbai, Mumbai University (India) in 2003 and Ph. D degree from IIT Roorkee in 2012. Presently, he is a faculty member in the Electrical Engineering Department at S.G.S Institute of Technology \& Science, Indore (M.P) - India. His research interests include Power System Restructuring, Power System Operations and Control, Energy management system, Distributed Generation, Renewable Energy Sources. 